\title{
Fault Diagnosis for Multi-energy Flows of Energy Internet: Framework and Prospects
}

\author{
Tao Wang, Jun Wang, Zhennan Fan \\ School of Electrical Engineering and Electronic Information \\ Sichuan Province Key Laboratory of Power Electronics \\ Energy-saving Technologies \& Equipment and Key Laboratory of \\ Fluid and Power Machinery, Ministry of Education \\ Xihua University \\ Chengdu, P. R. China \\ Tianlei Zang \\ State Key Laboratory of Security Control and Simulation of Power \\ Systems and Large Scale Generation Equipment, Department of \\ Electrical Engineering \\ Tsinghua University \\ Beijing, P. R. China
}

\begin{abstract}
Energy Internet (EI) is an inevitable development trend of energy systems under the background of technology development, environmental pressure and energy transition. Multi-energy flow coupling is one of the key characteristics of the EI, which enhances the interoperability of different types of energy flows while consequently increases the probability of cascading failures. Therefore it is of great significance to study the multi-energy flow fault diagnosis of the EI to ensure its safe and stable operation as well as the continuous energy supply. This paper introduces the concept of multi-energy flow cascading fault of the EI for the first time. The energy internet framework for multi-energy flow cascading fault diagnosis is firstly proposed, and then characteristics of various energy networks in the EI are analyzed from the perspective of fault diagnosis. Finally, future research prospects are discussed.
\end{abstract}

Keywords-energy Internet; fault diagnosis; multi-energy flow; multi-energy flow cascading fault; interoperability

\section{INTRODUCTION}

$\mathrm{W}$ ITH the great exploitation and fast depletion of fossil energy, the pressures caused by energy crisis and environmental pollution promote the transition of existing energy consumption forms. Therefore, how to use energy in a more efficient and clean way becomes a hot topic these days. Consequently, Energy Internet (EI) is proposed as one of the solutions and becoming the focus in the world ${ }^{[1]}$. The EI can be best described as a new type of energy network in which the energy can be freely transformed and transmitted in different kinds of forms among various energy systems (such as the power system, natural gas networks, cooling/heating system

This work was supported by the National Natural Science Foundation of China $(61703345,61472328,51607146)$

\author{
Xiaoguang Wei \\ School of Electrical Engineering \\ Southwest Jiaotong University \\ Chengdu, P. R. China \\ Mario J. Pérez-Jiménez \\ Research Groupon Natural Computing \\ Department of Computer Science and Artificial Intelligence \\ University of Sevilla \\ Sevilla, Spain \\ Tao Huang* \\ Department of Energy \\ Politecnico di Torino \\ Torino, Italy \\ Email: tao.huang@polito.it
}

and electrified traffic system) with a large number of distributed energy acquisition, storage devices and various types of load composition. The EI also uses many advanced technologies to support its interoperability among various enegy systems, including advanced monitoring and measuring, information and communication, intelligent management, big data and data analytics, etc.

The EI is the development and innovation stemmed from smart grids as a result of the deep integration of internet and energy systems, but it is more open with rich interconnection compared with smart grids. The openness and interconnection mainly appear in the following three points ${ }^{[1,2]}$. (a) The aim of the EI is to achieve the interoperability and interconnection of different energy systems and the focus of energy systems is changed from a single system to the energy system integration where multi-energy flow, such as electricity, cooling/heating, gas, electric transportation are considered. (b) The information technology is changed from the information and communication technology (ICT) to the Internet of Things (IoT). The general idea is to use the concepts in the internet to reform the existing energy industry and promote the formation of new industry status and new business models. (c) The EI emphasizes on the efficient and large-scaled use and consumption of renewable energy by the interoperable interconnection of multiple energy flows.

Thus, the EI is an integrated energy system which can realize comprehensive management and economic dispatch of different energy sources, including electricity, cooling/heating and gas et.. It can improve the energy efficiency both in terms of allocation and utilization, and provide solutions for balancing the needs of environment protection, economic development and energy security, etc. It is a key way to reach the goal of the optimization of energy infrastructure and the transition of energy systems from individual and separated ones to an integrated and interoperable on ${ }^{[3-5]}$. Integrated 
energy system under the background of energy interconnection has merits such as complementary advantages of different energy sources, improved energy efficiency, reduced cost, efficient utilization of renewable energy, etc.; however, the strong coupling of different energy systems also brings big challenges, such as the increased cascading risk ${ }^{[6,7]}$ for the safe and stable operation of the EI.

It is of important economic and practical significance to ensure the efficient and reliable operation of the EI to supply continuous and reliable energy ${ }^{[8]}$. However, due to various threats such as natural, accidental and even malicious, the EI inevitably has some potential dangers. Once faults occur, the deep coupled energy systems in the EI will be greatly impacted. If the accident is not handled timely and correctly, the fault will not only affect the system where it is originated but also propagate into other systems. It may eventually disturb the normal life of people and even bring serious economic losses. Thus, it is important to pay attention to the cascading failure diagnosis ${ }^{[9,10]}$ of the EI to provide promptly intervention. Multi-energy flow is one of the key characteristics of the EI, which means that there are inter-conversion, coupling and interaction among many types of energy sources and consumptions. Therefore, the research of multi-energy flow cascading fault diagnosis is vital to quickly and accurately detect fault areas, identify fault sections, identify fault types and then restore the energy supply to ensure the continuous energy flows in the EI.
However, up to now, the research achievements focus on the framework, connotation and models, basic concepts, system composition, integration scheme, working mechanism and energy management of the $\mathrm{EI}^{[2]}$. The safe and stable operation of the EI, especially the fault diagnosis, is rarely discussed. Thus, this paper proposes a conceptual framework and future research aspects about the multi-energy flow cascading fault diagnosis of the EI.

The remainder of this paper is organized as follows. Section II presents the conceptual framework and describes its important components and key links. Section III analyses the characteristics of each energy network in the EI from the point of view of fault diagnosis while section IV gives the future research aspects. Finally, conclusions are drawn in section V.

\section{CONCEPTUAL FRAMEWORK}

To analyse the fault diagnosis characteristics of different networks in the EI, this section firstly proposes the conceptual framework of the EI in considering multi-energy flow cascading fault diagnosis, as shown in Fig. 1. In the figure, the solid black, blue and yellow arrows represent physical connections, energy flows and chemical flows among different networks, respectively; the dashed red arrows represent information flows. The four types of flows are described in detail as follows.

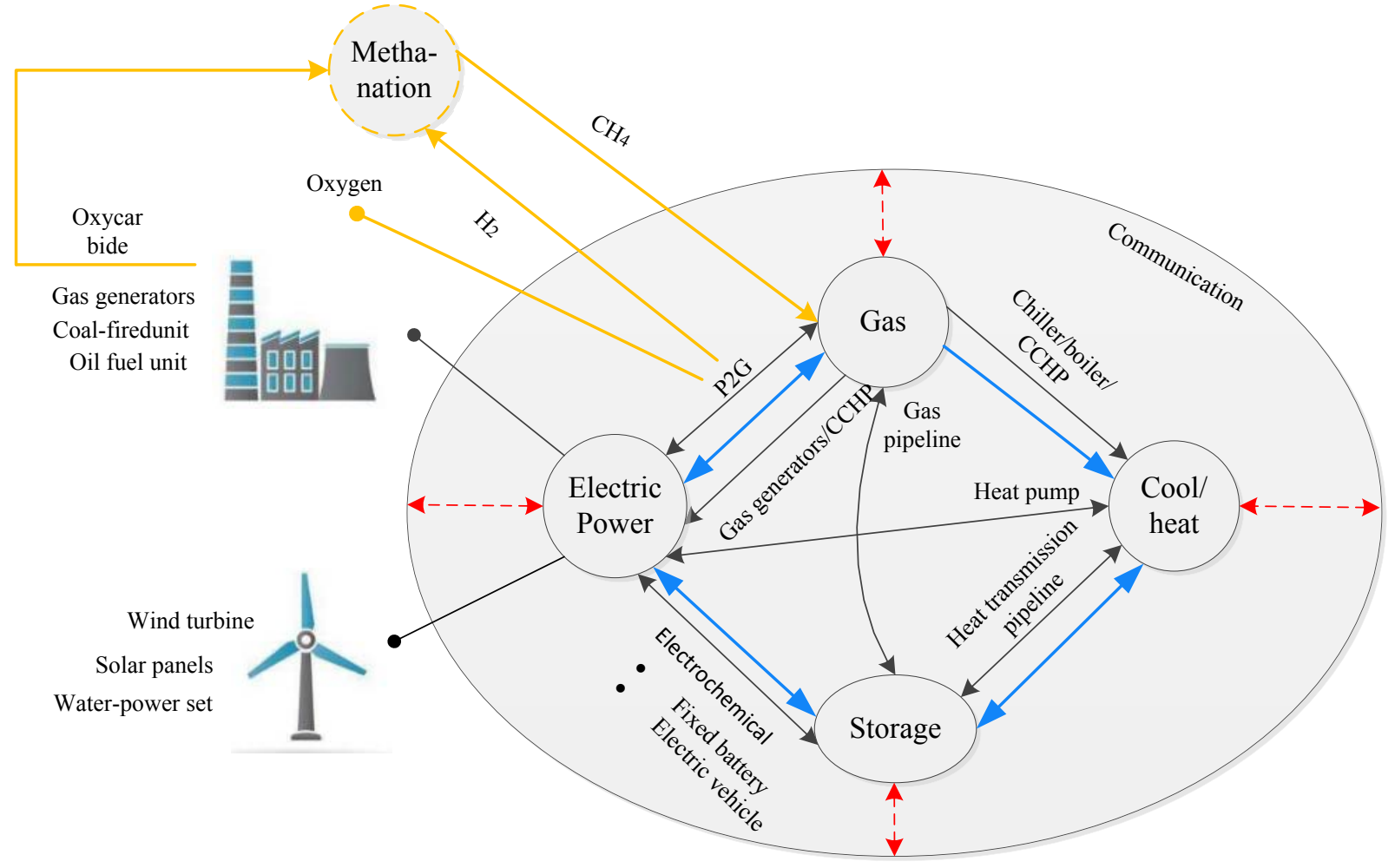

Fig. 1. Conceptual framework of the EI for multi-energy flow cascading fault diagnosis

The first type is the physical connections (solid black arrows) among different systems. In this paper, four energy systems are considered, namely electrical power systems, gas systems, cooling/heating systems and storage. To simplify the model of the EI, for power systems, renewable energy power generation and fossil fuel power generation are considered, 
where the renewable energy only considers wind, solar and hydro while the fossil fuel considers coal, gas and oil.

The five types of energy systems are coupled through three coupling sections which are used for energy production, system operation, and communication and information. For example, the power system and the gas system are coupled by power to gas (P2G) devices, gas generators and combined cooling heating and power (CCHP) generators while the gas system and the cooling / heating system are coupled together by chillers, gas boilers and CHPP generators. It should be noted that the electric vehicles are regarded as mobile batteries and only their charging and discharging characteristics as energy storage are considered. Thus, the power system and the energy storage are coupled by fixed batteries and electric vehicles. The gas system and the cooling/heating system are coupled with the energy storage through the gas pipelines and heat transmission pipelines, respectively.

Coupling sections used for energy production and system operation implement the coupling and interaction of different energy flows (solid blue arrows). The energy flows include a bidirectional flow between the power system and the gas system, a unidirectional flow from the gas system to the cooling/heating system, and two-way flows between the storage and the power system, the gas system or the cooling/heating system, respectively. The heterogeneous energy flows among different systems are one of the fundamental characteristics of the EI, and there are complex coupling relationships and different time scales ${ }^{[2-3]}$ that needs to be considered when modeling the different energy flows as a whole, as shown in Fig. 2. Thus, the multi-energy flow cascading fault diagnosis of the EI is more complicated and difficult than a single power flow cascading fault diagnosis in smart grids or others alike. Consequently, the conventional methods for modeling, analyzing and fault diagnosing of the traditional single energy flow system are unable to be used directly for such a complex system. Therefore, it is of great academic and engineering value to conduct research works on the multi-energy flow cascading fault diagnosis under the framework of energy internet.

In addition, there are a large number of information and communication coupling sections which cybernetically integrate multiple types of energy systems together by the ICT. The ICT in the EI is used for sharing information and coordinating the operation among various energy systems as well as providing measurements and data for the multi-energy flow cascading fault diagnosis. In this paper, the information flows (dashed red arrows) include two-way flows between the communication system and the power system, the gas system, the cooling/heating energy system or the storage, respectively.

Moreover, the proposed EI framework considers also chemical flows (solid yellow arrows) such as oxycarbide, hydrogen and methane. The combustion of fossil fuels will produce large amounts of oxycarbide which can be used for methanation with the hydrogen produced by electrolyzers, and then the acquired methane can be supplied into the gas system.

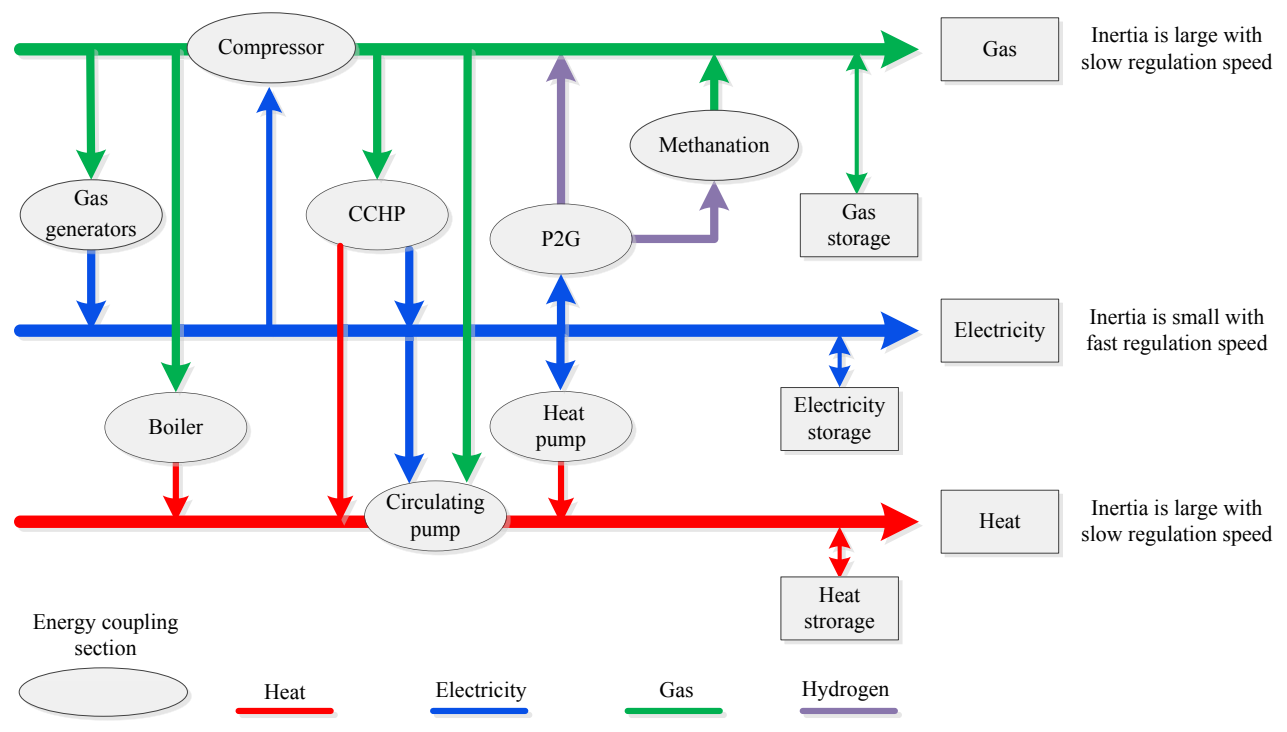

Fig. 2. Coupling relationships among multi-energy flows in the EI

\section{CHARACTERISTICS OF THE EI FROM THE FAULT DIAGNOSIS PERSPECTIVE}

This section firstly analyzes the characteristics of a single energy flow in each energy network (shown in Fig. 1) from the point of view of fault diagnosis and briefly summarizes the diagnosis methods and research status. Then, the characteristics of multi-energy flows in the EI are discussed. This paper considers the characteristics of single energy flow in the power system, gas system and the cooling/heating system from five respects, including research topics, diagnostic requirements, time characteristics, required detection and diagnosis method.

In power systems, the main faults are transverse faults, longitudinal faults, compound faults and natural disasters. The transverse faults include three-phase short-circuit, line to line faults, single-phase to earth faults and two-phase grounding faults. The longitudinal fault refers to the broken-line fault, 
such as single-phase disconnection, two-phase disconnection and three-phase disconnection. The compound fault means that there are at least two asymmetric faults occurring at different locations in a system. The natural disasters are the faults caused by natural disasters such as mud-rock flows, floods, rains, etc.

The research of power system fault diagnosis is mature and fruitful ${ }^{[6]}$. In TABLE 1 , the reasoning models mainly include Petri nets, expert systems, artificial neural networks, Bayesian networks, causal networks, fuzzy reasoning spiking neural $\mathrm{P}$ systems and so on. The information processing technology mainly refers to rough sets, fuzzy theory, data mining, information theory, multi agent systems and so on. The optimization technology refers to the diagnosis methods based on intelligent optimization algorithms, in which the diagnosis problem is abstracted as a $0-1$ programming problem. The primary data required for the detection of power system faults involves remote measurements and signals, including load flows and electrical measurements such as voltage, current and power over the lines, operational information of protective device, etc. As the failure process of power systems is very fast, the diagnosis process is complicated with a high requirement of diagnostic speeds.

At present, the fault diagnosis of gas systems focuses on the gas pipelines and other connected equipment. In the diagnosis of fault in the gas pipelines, the fundamentally used data are the pipe network parameters, such as pressure, flow rate, temperature etc. The important information used in diagnosing faults of gas generators are shaft vibration and thermal parameters, including performance parameters (such as efficiency and heat consumption rate, etc.) and measurable parameters (such as pressure, flow rate and temperature of air, effective power, etc). The analytic models in diagnosis method refer to state estimation, parameter estimation, equivalent space and Kalman filter, etc. The signal processing includes Fourier transform, wavelet transform, blind source separation and so on. Artificial intelligence mainly refers to the characteristic symptom diagnosis methods such as expert systems, fuzzy logic, neural networks. The speed of natural gas flows is slow compared with current in power systems, so the diagnosis of gas systems requires a relatively slow speed.
The fault diagnosis of cooling/heating systems focuses on heat transmission pipelines ${ }^{[11]}$ and heating circulating pumps. The diagnosis methods include technician checks based on empirical experiences and machine learning methods such as artificial neural networks, support vector machine, fuzzy theory, expert systems and rough sets. On the whole, the intelligent and automatic degree of cooling/heating system fault diagnosis is the lowest, and technicians still diagnose faults by looking, listening, smelling and toughing. That is to detect faults by listening to the sound in the heat transmission pipelines, running noise of the pumps, observing the flow rate, import and export pressure, as well as smelling burnt, and touching the pump body temperature. The cooling/heating energy flow is with large inertia and slow regulation, so it requires the minimum diagnostic speed among the different flows.

The multi-energy flow faults in the EI can be divided into three catalogues: (1) failures independently occur within an individual energy system and the coupling sections are not affected; (2) failures occur in an individual energy system and the coupling sections are affected; (3) coupling sections fault. For the first class, the fault diagnosis methods of single energy flow system are still usable. However, for the second and third cases, the diagnosis methods of a single energy flow system is no longer practicable because the different energy networks are deeply integrated together by the coupling sections. Thus, for the fault diagnosis of the latter two, not only the interactions between each single energy system and its corresponding coupling sections but also the impacts and influences from the coupling sections on the energy systems at both ends should be modeled. The pairwise coupling relationships between power systems, gas systems, cooling/heating energy systems and the energy storage in the EI have been described in Section II. The major coupling sections include gas generators, CCHP generators, $\mathrm{P} 2 \mathrm{G}$ devices, chillers, gas boilers, gas pipelines, heat pumps, heat transmission lines, fixed batteries and electric vehicles. When modeling the multi-energy flow fault diagnosis of the EI, the inherent fault behaviours of the coupling sections used for energy production and the coupling fault characteristics as coupling sections should be considered together.

TABLE I. FAULT CHARACTERISTICS OF NETWORKS IN THE EI

\begin{tabular}{|c|c|c|c|c|c|}
\hline $\begin{array}{c}\text { Energy } \\
\text { Network }\end{array}$ & Research Topics & $\begin{array}{c}\text { Time } \\
\text { Characteristics }\end{array}$ & $\begin{array}{c}\text { Diagnostic } \\
\text { Requirement }\end{array}$ & Required Data & Diagnosis Method \\
\hline $\begin{array}{c}\text { Electrical } \\
\text { power }\end{array}$ & $\begin{array}{c}\text { Transverse faults } \\
\text { Longitudinal fault } \\
\text { Complex faults } \\
\text { Natural disaster }\end{array}$ & $\begin{array}{c}\text { Smallest inertia } \\
\text { Fastest regulation } \\
\text { speed }\end{array}$ & Highest for speed & $\begin{array}{c}\text { Remote measurements } \\
\text { Remote signals }\end{array}$ & $\begin{array}{c}\text { Reasoning model } \\
\text { Information processing } \\
\text { Optimization }\end{array}$ \\
\hline Gas & $\begin{array}{c}\text { Gas pipelines } \\
\text { Gas equipment }\end{array}$ & $\begin{array}{c}\text { Middle inertia } \\
\text { speed }\end{array}$ & Middle for speed & $\begin{array}{c}\text { Pipe network Parameters } \\
\text { Shafting vibration and } \\
\text { thermal parameters }\end{array}$ & $\begin{array}{c}\text { Analytic model } \\
\text { Signal processing } \\
\text { Artificial intelligence }\end{array}$ \\
\hline Cool/heat & $\begin{array}{c}\text { Heat transmission } \\
\text { Pipelines } \\
\text { Heating Circulating } \\
\text { Pumps }\end{array}$ & $\begin{array}{c}\text { Biggest inertia } \\
\text { Slowest regulation } \\
\text { speed }\end{array}$ & Lowest for speed & Flow rate, pressure, \\
temperature, sound, etc. & Machine learning \\
\hline
\end{tabular}




\section{FUtURE RESEARCH ASPECTS}

To solve the challenges caused by the coupling and multi-time scales in the multi-energy flow cascading fault diagnosis, this section outlines the important future research works as follows:

(1) Fault characteristics analysis of multi-energy flow under multi-time scales. The fault characteristics are the most important foundation for building diagnosis models and designing of corresponding algorithms. The fault characteristics of different energy flows vary, thus several new fault characteristics will appear when the flows integrated together in a huge multi-layer dynamic complex system, i.e. energy internet. Most of the current research works just focus on the fault diagnosis of a single energy flow in each energy system. The study of multi-energy flow cascading fault diagnosis in an integrated energy system with several energy forms (such as electricity, gas, cool/heat) is yet very preliminary. The cascading fault characteristics of various energy flows in multi-time scale under the framework of multi-energy flow deep coupling are not thoroughly studied as well, not to mention the systematic study of multi-energy flow cascading fault diagnosis theory. Thus, research on cascading failure characteristics of multi-energy flows and the corresponding diagnosis theory of the EI is urgently needed.

(2) Coupling laws and models of different energy systems in the EI. With the advancement of the EI engineering and technology, the interconnection and coupling among power systems, gas systems, cooling/heating systems, electric transportation systems and even other new types of energy systems are inevitable. Therefore, it is essential to study the interoperability and coupling relationships among these energy systems and find out their coupling laws to reasonably and accurately build mathematical models of the entire EI. Up to now, the related research works are very scattered and in-depth studies are expected to grow in a short time.

(3) The designing and solving of multi-energy flow cascading fault diagnosis models across different time windows. The modeling methods of each system in the traditional electrical power systems, gas systems, cooling/heating energy systems before the stage of the EI are mature and abundant. The power grids follow the electromagnetic laws and are statically represented by the power flow models, where the main variables for the fault diagnosis include the voltage amplitude and angle, active/reactive power flows over lines and the active/reactive power injection/withdrawal of nodes and so on ${ }^{[2]}$. The cooling/heating energy systems follow the law of fluid mechanics and thermodynamics. The models include hydraulic and thermodynamic theories, where the main variables are pressure, flow, temperature and so on so forth ${ }^{[2]}$. The gas networks follow the law of fluid mechanics, and the main variables are pressure, flow and so on ${ }^{[2]}$. In a short time frame different energy networks are weakly coupled, while in a long time frame they are strongly coupled. The coupling relations between different flows show strong non-linear characteristics, thus complex systems solution, such as multi-agent systems can be considered. In addition, as the different energy systems are relatively independent with coupling relations in short time, the partition characteristics of membrane computing can be introduced to model the features of the EI.

\section{CONCLUSION}

The energy internet as one of the promising approaches to address the energy transition and sustainability through multi-energy flow coupling is attracting increasing attentions. However, as the dependence and entanglement of different energy systems are gradually getting enhanced, faults due to the interoperability can lead to a greater loss. Therefore, the study of multi-energy flow fault diagnosis of the EI is promptly needed.

In this paper, we propose a conceptual framework for investigating the multi-energy flow cascading fault diagnosis, and analyze the characteristics of various energy networks in the EI from the perspective of fault diagnosis. Finally, future research prospects are outlined.

\section{REFERENCES}

[1] H. Sun, Q. Guo, and Z. Pan, "Energy internet: concept, architecture and frontier outlook," Automation of Electric Power Systems, vol. 39, no. 19, pp.1-8, 2015.

[2] J. Wu, "Drivers and state-of-the art of integrated energy systems in Europe," Automation of Electric Power Systems, vol. 40, no. 5, pp. 1-7, 2016.

[3] H. Sun, Z. Guang, and Q. Guo, "Energy management for multi-energy flow: challenge and prospects," Automation of Electric Power Systems, vol. 40, no. 15, pp. 1-8, 2016.

[4] S. Ci, "Energy informatization and internet-based management and its applications in distributed energy storage system," Proceedings of the CSEE,vol. 35, no. 14, pp. 3643-3648, 2015.

[5] M. Zeng, Y.Yang, and Y. Li, et al, "The preliminary research for key operation mode and technologies of electrical power system with renewable energy sources under energy internet," Proceedings of the CSEE, vol. 36, no. 3, pp.681-691, 2016.

[6] Y. Cai, Y. Cao, and Y. Li, et al, "Cascading failure analysis considering interaction between power grids and communication networks", IEEE Transactions on Smart Grid, vol. 7, no. 1, pp. 530-538, 2016.

[7] A. Estebsari, E. Pons, and T. Huang, at al, "Techno-economic impacts of automatic undervoltage load shedding under emergency," Electric Power Systems Research, vol. 131, pp.168-177, 2016.

[8] A. Nurzhan Zhumabekuly, and S. Davor, "Security and privacy in decentralized energy trading through multi-signatures, blockchain and anonymous messaging streams," IEEE Transactions on Dependable and Secure Computing, vol. 13, no. 5, pp. 630-644, 2016.

[9] E. Bompard, A. Estebsari, and T. Huang, et al, "A framework for analyzing cascading failure in large interconnected power systems: A post-contingency evolution simulator," International Journal of Electrical Power and Energy Systems, vol. 81, pp. 12-21, 2016.

[10] T. Huang, S.L. Voronca, and A.A.Purcarea, et al, "Analysis of chain of events in major historic power outages," Advances in Electrical and Computer Engineering, vol. 14, no. 3, pp.63-70, 2014.

[11] Z. Li, W. Wu, and M. Shahidehpour, et al, "Combined heat and power dispatch considering pipeline energy storage of district heating network," IEEE Tran. Sustainable Energy, vol. 7, no. 1, pp. 12-22, 2016. 